\title{
Review
}

\section{CARD9 as a potential therapeutic target in lung cancer}

\author{
Ruanmei Sheng ${ }^{1,2}$, Zhiwen Yang ${ }^{3 *}$ \\ ${ }^{1}$ Department of Emergency Medicine and Intensive Care, Shanghai Songjiang District Central Hospital, 201600 \\ Shanghai, China, ${ }^{2}$ Department of Emergency Medicine and Intensive Care, Shanghai Songjiang Clinical Medical College \\ of Nanjing Medical University, 201600 Shanghai, China, ${ }^{3}$ Department of Pharmacy, Shanghai Songjiang District Central \\ Hospital, 201600 Shanghai, China
}

\section{TABLE OF CONTENTS}

\author{
1. Abstract \\ 2. Introduction \\ 3. CARD9 as a central integrator of immune response \\ 4. Clinical significance of CARD9 in lung cancer \\ 5. Preclinical studies of CARD9 in lung cancer \\ 6. CARD9 as a potential therapeutic target in lung cancer \\ 7. Conclusions \\ 8. Author contributions \\ 9. Ethics approval and consent to participate \\ 10. Acknowledgment \\ 11. Funding \\ 12. Conflict of interest \\ 13. References
}

\section{Abstract}

Caspase recruitment domain-containing protein 9 (CARD9) is highly expressed in myeloid cells and has been identified as a central regulator of innate immunity. Increasingly, studies demonstrate that CARD9 also plays a critical role in the development of lung cancer. This review focuses on the clinical significance and potential molecular mechanisms that CARD9 plays in lung cancer.

\section{Introduction}

Lung cancer remains a challenging disease largely due to its high mortality and morbidity. Data gathered in the USA indicate that in 2018 approximately 230,000 new cases of lung cancer were diagnosed and that this accounted for approximately $13 \%$ of all cancer cases [1-3]. Despite great advances in effective prevention, early diagnostic detection, and more effective therapeutics, many patients still experience disease recurrence without significant improvements in survival. The current therapeutic options for lung cancer include radiation, chemotherapy, and surgery. Each of these approaches has limitations, and patient prognosis remains dismal. Specifically, the 5-year survival rate for lung cancer is estimated at $18 \%$, and lung cancer is responsible for approximately $25 \%$ of all cancer-related deaths,

more than breast, colorectal, and prostate cancer [4, 5]. Therefore, it is critical to further explore molecular mechanisms that could improve diagnosis and therapy for lung cancer patients.

Emerging studies have emphasized the critical role of the tumor microenvironment (TME) in tumor progression. Myeloid-derived suppressor cells (MDSCs), a heterogenous group of immune cells derived from myeloid lineage, attenuate host immune responses through production of suppressive components within the TME [6-8]. In the context of the tumor, MDSCs can facilitate tumor escape, abnormally activate tumor cells, and suppress $\mathrm{T}$ cell function by increasing levels of reactive oxygen species, arginase, and nitric oxide synthase production [6, 8]. Additionally, MDSCs contribute to tumor angiogenesis, tumor cell invasion, and premetastatic niche formation through activation of non-immunologic mechanisms [9, 10].

It is clear that understanding MDSC biology is critical for improving clinical prognosis and improved treatment strategies in lung cancer patients [11]. Interestingly, CARD9 is highly expressed in MDSCs and exhibits a central role in regulating innate immunity [1]. Recently, it was reported that CARD9 function in MDSCs might drive a novel mechanism of lung cancer development [1, 12, 13]. This review focuses on our current understanding of 


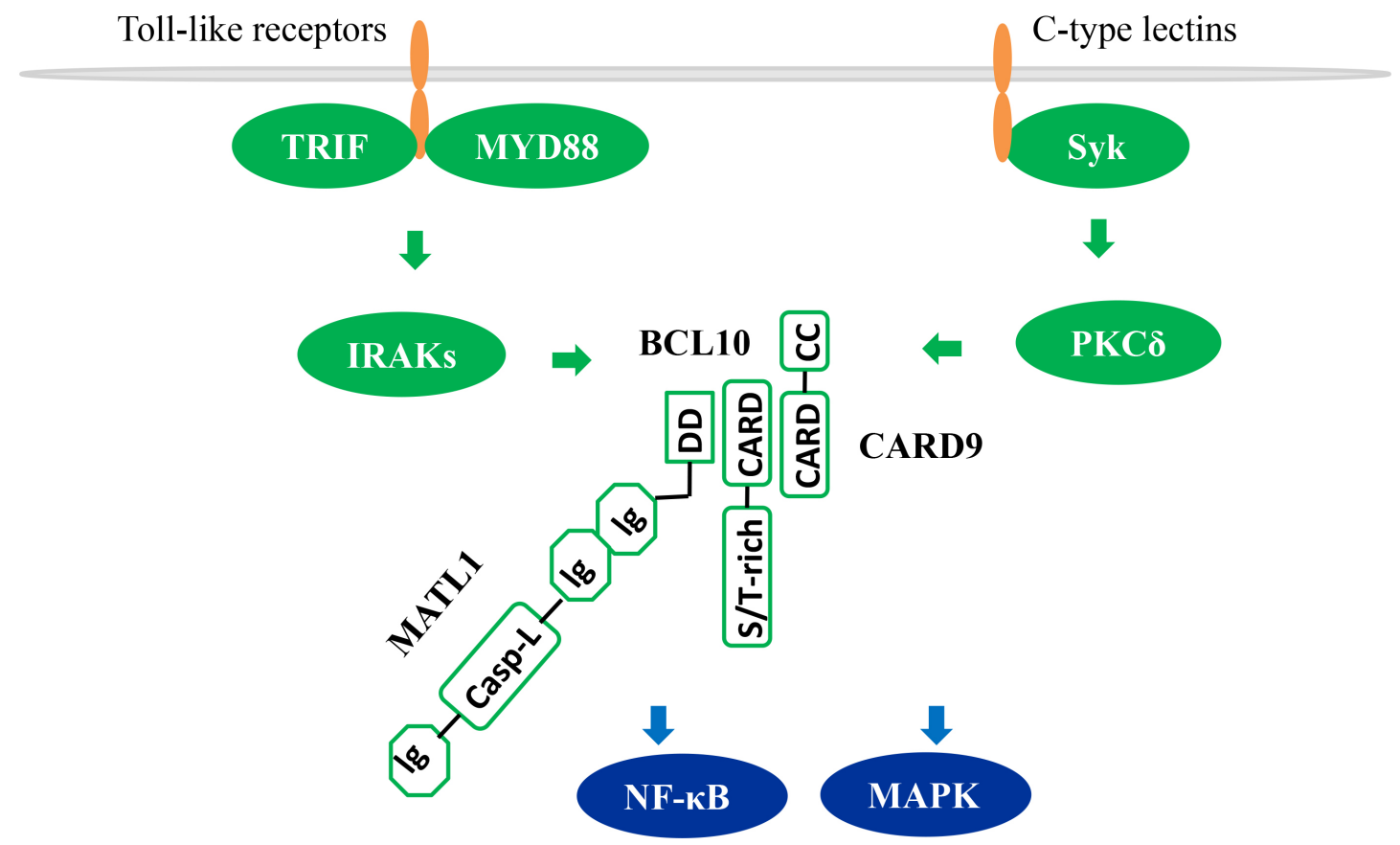

Fig. 1. CARD9-dependent NF- $\kappa$ B and p38 activation in myeloid cells.

CARD9 function in lung cancer and discusses CARD9 clinical significance, its molecular mechanism of action, as well as its potential as a therapeutic target.

\section{CARD9 as a central integrator of immune response}

CARD9 is highly expressed in myeloid cells especially macrophages, dendritic cells, and MDSCs [14-16]. CARD9 is known as the downstream effector molecule of the pattern recognition receptors including Toll-like receptors and C-type Lectins. Upon activation of these receptors, CARD9 triggers activation NF- $\kappa \mathrm{B}$ and/or mitogenactivated protein kinases (MAPK) signaling in myeloid cells (Fig. 1). This leads to activation of an inflammatory cytokine cascade that defends against microbial invasion, especially in fungal infection [14-16]. These findings have resulted in a consensus view that CARD9 functions as a central integrator of innate and adaptive immunity.

Through its myeloid cell functions, CARD9 has emerged as an essential player in tumor initiation and progression. We previously summarized the clinical significance of CARD9 in a variety of tumor types (Table 1) including hepatocellular carcinoma, intestinal carcinoma, gastric carcinoma, kidney carcinoma, and malignant pleural effusion [16, 17]. These studies suggest that high CARD9 expression is associated with tumor progression and poor survival rate [18-22]. However, preclinical studies in tumor-prone mouse models indicate that CARD9 plays a dual role in the carcinogenic process as it exhibits both a pro-tumor and/or anti-tumor activity [23-26]. Specifi- cally, CARD9 promoted tumor progression in kidney and intestinal cancer by activating NF- $\kappa \mathrm{B}$ and STAT3 signaling [23, 24]. Conversely, CARD9 inhibited tumor progression in leucocythemia and melanoma by both promoting tumor antigen-specific CD8 ${ }^{+}$cytotoxic T lymphocyte (CTL) cross-presentation and induction of cancer cell apoptosis $[25,26]$. This paradox may account for the cellular distribution of CARD9, as CARD9 expression in tumor tissues was found in tumor-infiltrating macrophages, but not tumor cells themselves [16, 17].

Macrophages are a prominent constituent of the tumor microenvironment where they can either promote, or suppress, tumor growth and metastasis depending on their biological state [27-29]. Further, CARD9-induced tumorigenesis was reported in a small cohort of patients, but exact evaluation in a large patient cohort is likely needed to confirm these findings.

\section{Clinical significance of CARD9 in lung cancer}

A recent study conducted by Qu et al. [1] studied 31 patients diagnosed with lung cancer. To address the relationship between CARD9 and MDSCs in these patients, CARD9 expression level and types of cells expressing CARD9 in lung tissues were examined. Compared with normal tissues, expression of CARD9 was significantly unregulated in both tumor and paracancerous tissues. The study documented that CARD9 was principally expressed in myeloid cells resident within the tumor tissue, but not tumor cells themselves. Furthermore, an increased popu- 
Table 1. The preclinical studies of CARD9 in tumor.

\begin{tabular}{lccc}
\hline Gene & Tumor & Function & Mechanisms \\
\hline CARD9 & Intestine & Pro-tumor & IL-1 $\beta$, IL-17A, IL-22, STAT3 \\
\hline CARD9 & Intestine & Pro-tumor & IL6, G-CSF, RANTES, T-cell, macrophage \\
\hline CARD9 & Intestine & Pro-tumor & IL-12, IL-10, IL-1 $\alpha$, TGF- $\beta$, NF-kB, M2 macrophage \\
\hline CARD9 & Kidney & Pro-tumor & Card9 linked pVHL to NF-kB biology \\
\hline CARD9 & Kidney & Pro-tumor & Card9-induced JNK hyper-activation \\
\hline CARD9 & Melanoma & Anti-tumor & Dectin-1-mediated cross-priming of CD8+ CTLs \\
\hline CARD9 & Leucocythemia & Anti-tumor & leukemic cell apoptosis \\
\hline
\end{tabular}

lation of MDSCs was observed in larger tumors and this was closely associated with higher expression of CARD9. Moreover, clinical pathology data indicated that CARD9 expression was positively correlated with tumor size [1]. In sum, this study provides a framework for understanding the contribution of CARD9 to lung cancer development in humans.

Miwa and colleagues reported that CARD9 served as a prognostic marker of poor outcome in lung cancer patients [13]. In this investigation, a total of 74 lung cancer patients diagnosed with adenocarcinoma were enrolled. Of these, 24 patients harbored CARD9-low expression, and the remining 50 cases were characterized as CARD9-high expression. Clinicopathologic analysis demonstrated that CARD9 expression was positively associated with poor overall survival, but not vascular and lymphatic invasion. This implicates CARD9 expression as an independent prognostic factor in lung cancer patients. Of note, in contrast to other studies that documented CARD9 expression uniquely in MDSCs [1], CARD9 was found to be expressed in adenocarcinoma cells in this study.

Pan and co-workers also identified CARD9 as a potential prognostic indicator in lung cancer [30]. Contrary to previous findings [1, 13], CARD9 expression was reduced in lung cancer and exhibited anti-tumor activity in this study. A total of 94 cases diagnosed with non-small cell lung cancer were studied in this report and CARD9 expression, at both protein and mRNA levels, indicated a clear decrease in cancer tissue in comparison with adjacent normal tissues. Clinicopathologic analysis indicated that CARD9 was negatively correlated with lymph node metastasis, $\mathrm{T}$ category, histological differentiation, tumor node metastasis (TNM) stage, and remote metastasis. Low CARD9 expression was associated with poor overall survival and progression-free survival of patients. Additionally, CARD9 in clinical specimens was found to be expressed in both tumor cells and macrophages.

\section{Preclinical studies of CARD9 in lung cancer}

Two studies reported that CARD9 regulated lung cancer through the control of MDSCs activation and in- doleamine 2, 3-dioxygenase (IDO) production [1, 12]. In the Lewis lung cancer mouse model, CARD9 deletion induced tumor cell proliferation, increased tumor size and weight, and aggravated splenomegaly. The expression level of MDSCs-related functional genes in tumor tissues of CARD9-deficient mice, such as iNOS-2, Arg-1, S100A8/A9, GP91phox and IDO, was significantly increased compared with that of wildtype mice. This result suggested that CARD9-deficiency in the TME may impair anti-tumor immune response through the activation and accumulation of immunosuppressive MDSCs. MDSCs are identified as the main obstacle in anticancer immunity and immunotherapy [31-33]. To test the pro-tumor function of MDSCs in CARD9-deficient mice, anti-Gr-1 monoclonal antibody, which effectively removes MDSCs, was injected into mice. As expected, this antibody decreased the number of MDSCs and coordinately increased the number of tumor-associated CD8 ${ }^{+}$CTLs within the tumor of CARD9deficient mice. Additionally, CARD9 deficiency promoted MDSCs differentiation and enhanced the inhibitory effect of MDSCs on T cells. Importantly, depleting MDSCs using anti-Gr1 antibody can markedly decrease tumor volume and tumor weight in CARD9-/- mice. Finally, the tumor regulatory mechanism was studied using CARD9 ${ }^{-/-}$micederived MDSCs. This study found that the non-canonical $\mathrm{NF}-\kappa \mathrm{B}$ pathway in MDSCs was activated in CARD9-/mice and that this enhanced expression IDO. IDO, an enzyme responsible for metabolism of tryptophan and one of the most important immunoregulatory enzymes, was critical to tumor development by regulating $\mathrm{T}$ cells [34-36]. Thus, within the TME, CARD9 promoted tumor growth via activation of the CARD9/NF- $\kappa \mathrm{B} / \mathrm{IDO}$ signaling cascade in MDSCs (Fig. 2A).

CARD9 protein was also found to be expressed in lung cancer cell lines PC9, H1299, and HBE [30], suggesting its potential participation in tumorigenesis. In vitro experiments showed that CARD9 knockdown in PC9 cells significantly promoted cell proliferation, invasion, migration, and inhibited cell apoptosis. Furthermore, reduced CARD9 expression activated p38 and contributed to tumor development in a p38-dependent manner. Contrary to previous findings regarding CARD9-induced p38 activation [37-39], in this study CARD9 deficiency upregulated 


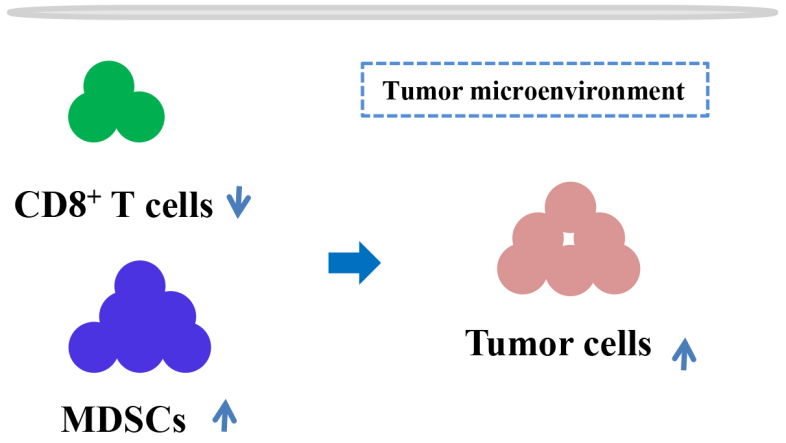

B PC9 tumor cells

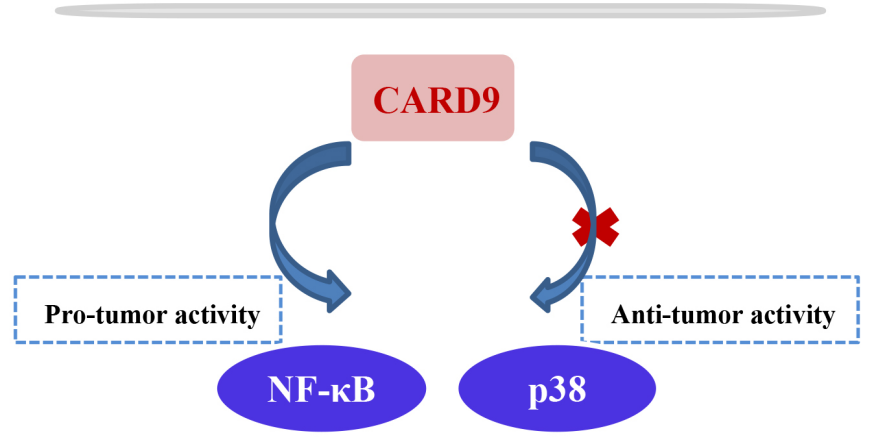

Fig. 2. The molecular mechanism of CARD9 in lung cancer. (A) CARD9-mediated immune response in the tumor microenvironment; (B) CARD9induced signalling pathway in the PC9 cell line.

p38 expression and the underlying molecular mechanism for this finding will need to elucidated in the future. Nevertheless, CARD9 has a significant anti-tumor response in lung tumors via downregulation of p38 signaling.

In this study, the investigators also showed that CARD9, in lung cancer cell lines, had a critical role in facilitating the progression of lung cancer [13]. CARD9 was upregulated in various lung cancer cell lines including PC9, A549 and II18. Furthermore, CARD9 was found to activate the canonical NF- $\kappa \mathrm{B}$ pathway in the PC9 cell line and, of note, CARD9 knockdown in PC9 cells inhibited cell proliferation, invasion, and migration. These findings are in contrast to previously outlined studies regarding CARD9 deficiency promoting tumor growth. This paradox may be attributable to CARD9 function two distinct molecular mechanisms in PC9 cells, specifically, CARD9-induced NF- $\kappa \mathrm{B}$ activation resulting in enhanced tumor cell growth versus CARD9-induced p38 deactivation resulting in inhibited cell growth (Fig. 2B). Nevertheless, this paradox suggests that CARD9 may play a dual role in lung tumor progression.

\section{CARD9 as a potential therapeutic target in lung cancer}

CARD9 expression in myeloid cells has been linked to tumor-related inflammation, tumor immune microenvironment, and intestinal microecology, suggesting that CARD9 may represent a potential target for anti-tumor immunotherapy [17].

Ganoderma lucidum polysaccharide (GLP), isolated from the fungus Ganoderma lucidum, serves as an effective anti-tumor agent that significantly inhibits tumor growth in the Lewis lung cancer mouse model. Moreover, GLP administration to these mice [40] can effectively suppress the accumulation of MDSCs in the spleen and tumor tissues, increase the percentage of $\mathrm{CD} 4^{+}$and $\mathrm{CD}^{+}$ $\mathrm{T}$ cells in the spleen, and reverse the attenuated expression of CARD9 and p65 in MDSCs. Furthermore, GLP can ele- vate expression of IDO in MDSCs, suggesting an activation of the CARD9/NF- $\kappa \mathrm{B} / \mathrm{IDO}$ signaling cascade in MDSCs within the TME. Collectively, targeting CARD9 with GLP can prevent lung cancer development by suppressing accumulation and differentiation of MDSCs in tumor-bearing mice.

$\beta$-glucan, a natural polysaccharide compound, binds to C-type lectin receptors and subsequently activates CARD9-dependent signaling [41]. $\beta$-glucan was found to reduce tumor burden with less vascular structure in the Lewis lung carcinoma mouse model [41]. Mechanistically, $\beta$-glucan converts tumor-associated macrophages into antitumor macrophages of the M1 phenotype via activation of the Dectin-1/CARD9 signaling pathway.

\section{Conclusions}

CARD9 is a central integrator of innate immunity in myeloid cells and plays a key role in lung cancer pathogenesis. However, some important questions regarding CARD9 remain unknown. First, it is unclear whether CARD9 is protective against lung cancer development. A total of three papers have been published that report on the clinical significance of CARD9 in lung cancer. Of these, two indicate CARD9 possesses pro-tumor activity, and one paper suggests anti-tumor activity. Of note, in vivo studies in mice support an anti-tumor function for CARD9 and this contradicts clinical data obtained from humans. This discrepancy may be due to high expression of CARD9 in patient samples because of the increased proportion of myeloid cells within the tumor [1]. Future studies will be needed to fully understand CARD9 pro-tumor versus antitumor functions using a large cohort of patients.

Second, the cellular distribution of CARD9 in lung cancer remains controversial; specifically at question is whether CARD9 is expressed in MDSCs and/or lung tumor cells. As previously reported [42-44], investigators documented that CARD9 expression was limited to tumor- 
infiltrating macrophages in colon cancer. In contrast, studies of oral squamous cell carcinoma [45] and renal cell carcinoma [44] revealed CARD9 expression within tumor cells. Others documented CARD9 expression, at both the protein and mRNA levels, is upregulated in various cultured lung cancer cell lines, suggesting that CARD9 is not only expressed in MDSCs but also in lung tumor cells. In contrast, some clinical data gathered from lung cancer patients clearly show that CARD9 was only expressed in MDSCs rather than in tumor cells [1].

Despite the uncertainties outlined in this review, the bulk of studies support an anti-tumor function for CARD9 in lung cancer. More studies, focused on the biological function of CARD9, will be required to better determine how MDSCs interact with tumor cells in a CARD9dependent mechanism.

\section{Author contributions}

RS-writing-original draft preparation; ZYwriting-review and editing. All authors have read and agreed to the published version of the manuscript.

\section{Ethics approval and consent to participate}

Not applicable.

\section{Acknowledgment}

Not applicable.

\section{Funding}

This research received no external funding.

\section{Conflict of interest}

The authors declare no conflict of interest.

\section{References}

[1] Qu J, Liu L, Xu Q, Ren J, Xu Z, Dou H, et al. CARD9 prevents lung cancer development by suppressing the expansion of myeloid-derived suppressor cells and IDO production. International Journal of Cancer. 2019; 145: 2225-2237.

[2] Pockley AG, Vaupel P, Multhoff G. NK cell-based therapeutics for lung cancer. Expert Opinion on Biological Therapy. 2020; 20: 23-33.

[3] Wang J, Li X, Chen H. Organoid models in lung regeneration and cancer. Cancer Letters. 2020; 475: 129-135.

[4] Altorki NK, Markowitz GJ, Gao D, Port JL, Saxena A, Stiles $\mathrm{B}$, et al. The lung microenvironment: an important regulator of tumour growth and metastasis. Nature Reviews Cancer. 2019; 19: 9-31.

[5] Zhang L, Zhu B, Zeng Y, Shen H, Zhang J, Wang X. Clinical lipidomics in understanding of lung cancer: Opportunity and challenge. Cancer Letters. 2020; 470: 75-83.
[6] Gabrilovich DI, Nagaraj S. Myeloid-derived suppressor cells as regulators of the immune system. Nature Reviews Immunology. 2009; 9: 162-174.

[7] Milette S, Fiset PO, Walsh LA, Spicer JD, Quail DF. The innate immune architecture of lung tumors and its implication in disease progression. The Journal of Pathology. 2019; 247: 589605.

[8] Srivastava MK, Andersson A, Zhu L, Harris-White M, Lee JM, Dubinett $\mathrm{S}$, et al. Myeloid suppressor cells and immune modulation in lung cancer. Immunotherapy. 2012; 4: 291-304.

[9] Ortiz ML, Lu L, Ramachandran I, Gabrilovich DI. MyeloidDerived Suppressor Cells in the Development of Lung Cancer. Cancer Immunology Research. 2014; 2: 50-58.

[10] Srivastava MK, Zhu L, Harris-White M, Kar UK, Huang M, Johnson MF, et al. Myeloid suppressor cell depletion augments antitumor activity in lung cancer. PLoS ONE. 2012; 7: e40677.

[11] Yang Z, Guo J, Weng L, Tang W, Jin S, Ma W. Myeloid-derived suppressor cells-new and exciting players in lung cancer. Journal of Hematology \& Oncology. 2020; 13: 10.

[12] Wu X, Li F, Deng Y, Fan X. Analysis of the control mechanism of lung cancer of caspase recruitment domain-containing protein 9 and myeloid-derived suppressor cell in Lewis lung cancer mice model. Saudi Journal of Biological Sciences. 2019; 26: 20372042.

[13] Miwa N, Nagano T, Jimbo N, Dokuni R, Kiriu T, Mimura C, et al. Caspase Recruitment Domain-Containing Protein 9 Expression is a Novel Prognostic Factor for Lung Adenocarcinoma. OncoTargets and Therapy. 2020; 13: 9005-9013.

[14] Yang ZW, Weng CZ, Wang J, Xu P. The role of Card9 overexpression in peripheral blood mononuclear cells from patients with aseptic acute pancreatitis. Journal of Cellular and Molecular Medicine. 2016; 20: 441-449.

[15] Yang ZW, Meng XX, Zhang C, Xu P. CARD9 gene silencing with siRNA protects rats against severe acute pancreatitis: CARD9-dependent NF-kappaB and P38MAPKs pathway. Journal of Cellular and Molecular Medicine. 2017; 21; 1085-1093.

[16] Zhong XM, Chen B, Yang L, Yang Z. Molecular and physiological roles of the adaptor protein CARD9 in immunity. Cell Death \& Disease. 2018; 9: 52.

[17] Zhong XM, Chen B, Yang L, Yang Z. Card9 as a critical regulator of tumor development. Cancer Letters. 2019; 451: 150-155.

[18] Zekri AR, El-Kassas M, Saad Y, Bahnassy A, El-Din HK, Darweesh SK, et al. Caspase recruitment domains. New potential markers for diagnosis of hepatocellular carcinoma associated with HCV in Egyptian patients. Annals of Hepatology. 2013; 12: 774-781.

[19] Yang M, Shao JH, Miao YJ, Cui W, Qi YF, Han JH, et al. Tumor cell-activated CARD9 signaling contributes to metastasisassociated macrophage polarization. Cell Death \& Differentiation. 2014; 21: 1290-1302.

[20] Nakamura S, Nakamura S, Matsumoto T, Yada S, Hirahashi M, Suekane $\mathrm{H}$, et al. Overexpression of caspase recruitment domain (CARD) membrane-associated guanylate kinase 1 (CARMA1) and CARD9 in primary gastric B-cell lymphoma. Cancer. 2005; 104: 1885-1893.

[21] Tan W, Hildebrandt MAT, Pu X, Huang M, Lin J, Matin SF, et al. Role of Inflammatory Related Gene Expression in Clear Cell Renal Cell Carcinoma Development and Clinical Outcomes. Journal of Urology. 2011; 186: 2071-2077.

[22] Li H, Tang Z, Zhu H, Ge H, Cui S, Jiang W. Proteomic study of benign and malignant pleural effusion. Journal of Cancer Research and Clinical Oncology. 2016; 142: 1191-1200.

[23] Bergmann H, Roth S, Pechloff K, Kiss EA, Kuhn S, Heikenwälder M, et al. Card9-dependent IL-1beta regulates IL-22 production from group 3 innate lymphoid cells and promotes colitisassociated cancer. European Journal of Immunology. 2017; 47: 1342-1353.

[24] Yang H, Minamishima YA, Yan Q, Schlisio S, Ebert BL, Zhang $\mathrm{X}$, et al. pVHL acts as an adaptor to promote the inhibitory phos- 
phorylation of the NF-kappaB agonist Card9 by CK2. Molecular Cell. 2007; 28: 15-27.

[25] Haas T, Heidegger S, Wintges A, Bscheider M, Bek S, Fischer JC, et al. Card9 controls Dectin-1-induced T-cell cytotoxicity and tumor growth in mice. European Journal of Immunology. 2017; 47: 872-879.

[26] Yang J, Chai L, Gao C, Fowles TC, Alipio Z, Dang H, et al. SALL4 is a key regulator of survival and apoptosis in human leukemic cells. Blood. 2008; 112: 805-813.

[27] Pan Y, Yu Y, Wang X, Zhang T. Tumor-Associated Macrophages in Tumor Immunity. Frontiers in Immunology. 2020; 11: 583084.

[28] Xu F, Wei Y, Tang Z, Liu B, Dong J. Tumorassociated macrophages in lung cancer: Friend or foe? (Review). Molecular Medicine Reports. 2020; 22: 4107-4115.

[29] Zhou Y, Fei M, Zhang G, Liang WC, Lin W, Wu Y, et al. Blockade of the Phagocytic Receptor MerTK on Tumor-Associated Macrophages Enhances P2X7R-Dependent STING Activation by Tumor-Derived cGAMP. Immunity. 2020; 52: 357-373.e9.

[30] Pan L, Tan Y, Wang B, Qiu W, Yin Y, Ge H, et al. Caspase Recruitment Domain Containing Protein 9 Suppresses NonSmall Cell Lung Cancer Proliferation and Invasion via Inhibiting MAPK/p38 Pathway. Cancer Research and Treatment. 2020; 52: 867-885.

[31] Greene S, Robbins Y, Mydlarz WK, Huynh AP, Schmitt NC, Friedman J, et al. Inhibition of MDSC Trafficking with SX682, a CXCR1/2 Inhibitor, Enhances NK-Cell Immunotherapy in Head and Neck Cancer Models. Clinical Cancer Research. 2020; 26: 1420-1431.

[32] Li BH, Garstka MA, Li ZF. Chemokines and their receptors promoting the recruitment of myeloid-derived suppressor cells into the tumor. Molecular Immunology. 2020; 117: 201-215.

[33] Kim W, Chu TH, Nienhüser H, Jiang Z, Del Portillo A, Remotti HE, et al. PD-1 Signaling Promotes Tumor-Infiltrating MyeloidDerived Suppressor Cells and Gastric Tumorigenesis in Mice. Gastroenterology. 2021; 160: 781-796.

[34] Campesato LF, Budhu S, Tchaicha J, Weng CH, Gigoux M, Cohen IJ, et al. Blockade of the AHR restricts a Treg-macrophage suppressive axis induced by L-Kynurenine. Nature Communications. 2020; 11: 4011.

[35] Zhai L, Bell A, Ladomersky E, Lauing KL, Bollu L, Sosman JA, et al. Immunosuppressive IDO in Cancer: Mechanisms of Action, Animal Models, and Targeting Strategies. Frontiers in Immunology. 2020; 11: 1185.

[36] Zhou C, Zhang Y, Yan R, Huang L, Mellor AL, Yang Y, et al.
Exosome-derived miR-142-5p remodels lymphatic vessels and induces IDO to promote immune privilege in the tumour microenvironment. Cell Death \& Differentiation. 2021; 28: 715729.

[37] Goodridge HS, Shimada T, Wolf AJ, Hsu YM, Becker CA, Lin $\mathrm{X}$, et al. Differential Use of CARD9 by Dectin- 1 in Macrophages and Dendritic Cells. The Journal of Immunology. 2009; 182: 1146-1154.

[38] Hara H, Saito T. CARD9 versus CARMA1 in innate and adaptive immunity. Trends in Immunology. 2009; 30: 234-242.

[39] Colonna M. All roads lead to CARD9. Nature Immunology. 2007; 8: 554-555.

[40] Wang Y, Fan X, Wu X. Ganoderma lucidum polysaccharide (GLP) enhances antitumor immune response by regulating differentiation and inhibition of MDSCs via a CARD9NF-kappaB-IDO pathway. Bioscience Reports. 2020; 40: BSR20201170.

[41] Liu M, Luo F. Ding C, Albeituni S, Hu X, Ma Y, et al. Dectin-1 Activation by a Natural Product beta-Glucan Converts Immunosuppressive Macrophages into an M1-like Phenotype. Journal of Immunology. 2015; 195: 5055-5065.

[42] Malik A, Sharma D, Malireddi RKS, Guy CS, Chang TC, Olsen SR, et al. SYK-CARD9 Signaling Axis Promotes Gut Fungi-Mediated Inflammasome Activation to Restrict Colitis and Colon Cancer. Immunity. 2018; 49: 515-530.e5.

[43] Wang T, Fan C, Yao A, Xu X, Zheng G, You Y, et al. The Adaptor Protein CARD9 Protects against Colon Cancer by Restricting Mycobiota-Mediated Expansion of Myeloid-Derived Suppressor Cells. Immunity. 2018; 49: 504-514.e4.

[44] Zhong XM, Chen B, Liu M, Yang Z. The Role of Adaptor Protein CARD9 in Colitis-Associated Cancer. Molecular Therapy Oncolytics. 2019; 15: 1-6.

[45] Ye LJ, Zhou XC, Yin XJ, Shang Y, Xiao Y, Jiang Y. et al. CARD9 downregulation suppresses the growth of oral squamous cell carcinoma by regulating NF-kappaB. Oral Diseases. 2019; 25: 1886-1896.

Keywords: CARD9; Lung cancer; Clinical significance; Molecular mechanism; Therapy target

Send correspondence to: Zhiwen Yang, Department of pharmacy, Shanghai Songjiang District Central Hospital, 201600 Shanghai, China, E-mail: yangzhiwen2009@sina.com 